\title{
VALIDACIÓN TÉCNICA DE INOCULANTES EN FRIJOL CON CEPAS DE Rhizobium EFICIENTES EN FIJACIÓN DE NITRÓGENO EN CENTROAMÉRICA ${ }^{1}$
}

\author{
Oscar Acuña ${ }^{2}$, Emigdio Rodríguez ${ }^{3}$, Aurelio Llano ${ }^{4}$, Vilma Ruth Calderón ${ }^{5}$, Guillermo Flores ${ }^{6}$, \\ Abelardo Viana ${ }^{7}$, Rogelio Lépiz 8
}

\begin{abstract}
RESUMEN
Validación técnica de inoculantes en frijol con cepas de Rhizobium eficientes en fijación de nitrógeno en Centroamérica. Se realizó un ensayo de validación técnica de inoculantes en frijol elaborado con cepas de Rhizobium eficientes en fijación biológica de nitrógeno, con el objetivo de recabar mayor información sobre la bondad de esta tecnología en la producción comercial de frijol. El ensayo regional se sembró en 45 sitios, distribuidos en Costa Rica (20), Panamá (10), Nicaragua (10) y El Salvador (5), durante 1998 y 1999. Se incluyeron cuatro tratamientos: 1 . Testigo absoluto; 2. Inoculado; 3. Fertilización intermedia e inoculación; 4. Fertilización recomendada (Fer Rec). El inoculante fue preparado y distribuido por la Universidad de Costa Rica; la variedad y la fertilización utilizada en los tratamientos tres y cuatro, fueron las recomendadas localmente. La parcela experimental varió de 50 a $100 \mathrm{~m}^{2}$ y se utilizó un diseño de bloques al azar, con tres repeticiones. Se cosecharon 39 ensayos y se registraron rendimientos de grano y costos de producción. El análisis de varianza por áreas de producción y países, utilizó el promedio por sitio; el análisis económico fue de presupuesto parcial. Los resultados mostraron lo siguiente: a) la respuesta del frijol a la inoculación con Rhizobium y/o fertilizantes, varió de un sitio a otro; b) el análisis regional mostró respuesta del rendimiento al uso de inoculante y en el $80 \%$ de los casos el análisis económico, seleccionó a la inoculación como redituable; c) a excepción de Panamá, el uso de la mitad del fertilizante recomendado más inoculación, fue superior al tratamiento con sólo inoculante.
\end{abstract}

\begin{abstract}
Technical evaluation of inoculants in common bean with efficient Rhizobium strains in nitrogen fixation in Central America. A technical evaluation trial was carried out to evaluate the use of inoculants in bean with efficient Rhizobium strains, in oder to obtain more information about the usefulness of this technology in the common bean production. The regional experiment was planted in 45 localities distributed in Costa Rica (20), Panama (10), Nicaragua (10) and El Salvador (5), during 1998 and 1999. The experiment included four treatments: 1. Control; 2. Inoculation; 3. Half of recommended fertilization plus inoculation; 4. Recommended fertilization (Fer Rec). The inoculant was prepared and distributed by the University of Costa Rica. The variety and the fertilization utilized in treatments 3 and 4, were the recommended in each locality. The experimental plot varied between 50 and $100 \mathrm{~m}^{2}$ and a randomized complete block design with three replications was used. Thirty-nine trials were harvested and the seed yield and production costs were registered. The analysis of variance by production areas and countries, used the yield average by location; the economic analysis used the partial budget methodology. The results showed that: a) the bean response to inoculation and/or fertilization, varied among places; b) the regional analysis indicated bean response to the inoculant utilization and in the $80 \%$ of the cases, the economic analysis selected the inoculation treatment as profit; c) with the exception of Panama, the use of half of recommended fertilization plus inoculation, was better than inoculation only.
\end{abstract}

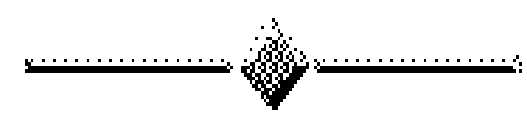

\section{INTRODUCCION}

En países como México, en los años 60's y 70's, se hicieron evaluaciones de inoculantes comerciales a ba- se de Rhizobium en frijol, con poco éxito. La baja o nula respuesta del frijol a la inoculación, se ha atribuido entre otras cosas a la ineficiencia y mala calidad de los inoculantes comerciales. Sin embargo se señala también

\footnotetext{
1 Investigación financiada parcialmente por el Programa Cooperativo Regional de Frijol para Centroamérica, México y El Caribe (PROFRIJOL).

2 Universidad de Costa Rica, San José, Costa Rica.

3 Instituto de Investigación Agropecuaria de Panamá, David, Panamá.

${ }^{4}$ Instituto Nicaragüense de Tecnología Agropecuaria, Managua, Nicaragua.

5 Centro de Tecnología Agropecuaria, San Salvador, El Salvador.

6 Ministerio de Agricultura y Ganadería, San José, Costa Rica.

7 Socioeconomía y actual Coordinador Regional de PROFRIJOL, Guatemala, Guatemala.

${ }^{8}$ Coordinador Regional de PROFRIJOL y actual Profesor Investigador Universidad de Guadalajara, México.
} 
que los factores del ambiente y aquellos relacionados con la planta y la bacteria, juegan un papel importante en el éxito de un inoculante (Graham et al., 1981; Graham, 1990; Ferrera et al., 1990; Trujillo, 1990).

Graham (1990) en su revisión de problemas en la nodulación y fijación de nitrógeno en frijol, consigna lo siguiente: a) existe una amplia variación entre genotipos en fijación simbiótica de $\mathrm{N}_{2}$ (de tres a $125 \mathrm{kh} / \mathrm{ha}$ ), donde los cultivares de hábito indeterminado trepador son más eficientes; b) hay un progreso en el desarrollo de variedades con alta capacidad en fijación biológica del nitrógeno, a través del mejoramiento genético utilizando métodos de selección recurrente; c) se han identificado cepas de Rhizobium como CIAT 640, RCR 3644, TAI 182 y C-5, altamente efectivas a través de un rango variable de condiciones de suelo y clima; d) los factores ambientales, como acidez (menor de 4,5), toxicidad de aluminio y manganeso y disponibilidad de fósforo en el suelo, temperatura ambiente y competencia con cepas nativas, afectan significativamente la capacidad de nodulación y fijación biológica del nitrógeno por el frijol.

Por su parte Ferrera et al., (1990) mencionan que la variedad de frijol juega un papel importante en el mecanismo de reconocimiento de la planta hacia la bacteria, mostrando mayor afinidad con ciertas cepas. También señalan diferencias en la capacidad de fijación entre variedades y la existencia de cierta preferencia de los cultivares de frijol por una fuente particular de nitrógeno. En un estudio con variedades de diferente hábito y utilizando $15 \mathrm{~N}$, encontraron diferencias en la fijación simbiótica del nitrógeno entre variedades: a) Bayomex de hábito I, fijó solamente $7,1 \mathrm{~kg} / \mathrm{ha}$ de nitrógeno; b) Ojo de Cabra 400 de hábito II, fijó 13,3kg/ha de nitrógeno; c) Criollo Tequesquinagua de hábito III, 106,5 $\mathrm{kg} / \mathrm{ha}$ de nitrógeno. En forma similar, en Bayomex el 4,87\% del nitrógeno total de la planta, procedió del nitrógeno fijado; en Ojo de Cabra 400, el 8,12\% y en Criollo Tequesquinagua, el 44,64\% del total de nitrógeno total utilizado por la planta, fue de origen simbiótico. Ferrera et al., (1990) mencionan también que a pesar de la influencia de los factores del medio ambiente, es posible encontrar cepas con cierta plasticidad, lo cual les permite adaptarse a más de un ambiente.

Ferrera y Almaráz (1996), mencionan que la selección de variedades de frijol y cepas de Rhizobium adaptadas a diversas condiciones es necesaria para incrementar la fijación de nitrógeno. En el desarrollo de esta biotecnología, es importante entender los factores ambientales y biológicos que afectan la simbiosis. Un conocimiento integral de la triada bacteria-planta-ambiente permitiría definir estrategias que conduzcan a la selección de variedades y cepas de Rhizobium con alta fijación de nitrógeno y tolerantes a diferentes factores del ambiente.

Rodríguez y Ferrera (1984), citados por Ferrera $e t$ al., (1990), realizaron una selección de cepas de Rhizobium en cuatro variedades de frijol de diferente hábito de crecimiento. Las variedades mostraron respuesta diferente a la inoculación con las diferentes cepas de Rhizobium: el 37,5\% de las cepas se comportó como efectivo en más de una variedad; el $26 \%$ fueron efectivas en dos variedades; el $10 \%$ se comportó como efectivo en fijación de nitrógeno en tres variedades; sólo la cepa. El 63 resultó ser efectiva en las cuatro variedades. Concluyeron que existe interespecificidad entre cepa y variedad de frijol, interacción que es necesario considerar en un programa de selección de cepas de Rhizobium y/o genotipos de frijol. El estudio muestra que algunas cepas pueden nodular y fijar $\mathrm{N}$ en forma eficiente en más de una variedad.

No obstante que en muchos experimentos de campo se ha encontrado que la aplicación de fertilizante nitrogenado al suelo disminuye la nodulación, algunos investigadores indican que la adición de nitrógeno en dosis bajas ( 5 a $15 \mathrm{~kg} / \mathrm{ha}$ ), promueve el desarrollo de los nódulos Graham y Halliday (1997), citados por Ferrera, et al., 1990). López et al., (1984), citados por Ferrera et al., (1990), en un estudio de tres cepas de Rhizobium con dosis crecientes de nitrógeno, encontraron que aunque el aumento de las dosis de nitrógeno redujo el número de nódulos, existió nodulación en las tres cepas aún en las dosis altas de nitrógeno. Sus resultados muestran que hay cepas de Rhizobium capaces de nodular en presencia de dosis altas de nitrógeno, por lo que es posible aportar al cultivo una fuente adicional de nitrógeno.

Acuña y Cordero (1989) encontraron que en suelos de baja fertilidad y alta acidez se obtuvo un efecto beneficioso sobre la nodulación cuando se fertilizó con fósforo y calcio. Se ha observado además que plantas dependientes de la fijación biológica de nitrógeno requieren de más fósforo que las que utilizan nitrógeno mineral. La aplicación de fertilizante fosfórico mejora la nodulación y la efectividad de la fijación (Rosas y Bliss, 1986; Morales, 1987).

Beck y Valdez (1994), encontraron diferencias en fijación biológica de nitrógeno entre genotipos de frijol, al ser evaluados en alta y baja disponibilidad de fósforo. Los genotipos mostraron habilidad contrastante para fijar nitrógeno; los resultados se dieron en alta y baja disponibilidad de fósforo. Encontraron que algunos genotipos 
mostraron altos niveles de fijación de $\mathrm{N}$, contradiciendo la afirmación de que el frijol es un pobre fijador. Algunos genotipos fueron marcadamente limitados en fijar $\mathrm{N}$ en condiciones de deficiencia de fósforo; este resultado enfatiza la necesidad de realizar la selección de genotipos en alta y baja disponibilidad de fósforo.

En la Sierra Peruana la deficiencia de nitrógeno en los suelos, es una de las causas principales de los bajos rendimientos en cultivos como maíz y frijol. Una alternativa de bajo costo para reducir este problema, es el nitrógeno fijado por la leguminosa. Con este propósito, el Proyecto de Frijol del CIAT para la Zona Andina (PROFRIZA), realizó trabajos principalmente en selección de cepas efectivas de Rhizobium con amplio rango de adaptación y con capacidad de competir con las cepas nativas. El trabajo dio énfasis a la evaluación de cepas (siete nativas y siete del CIAT) en campos de agricultores en la región de Cajamarca, en la Sierra Norte de Perú. Después de cuatro campañas agrícolas, en 1991 se seleccionaron cuatro cepas con las características deseadas: CIAT 701, Cusco 10, CIAT 2 y Cajamarca 13. En los ensayos de campo, se lograron incrementos del $22 \%$ en la producción de frijol y del $42 \%$ en la producción de maíz. Adicionalmente, en 1991 y 1992, se produjo inoculante comercial, componente que fue bien aceptado por los productores (Pineda, 1992).

De acuerdo con Hernández et al. (1994), en Cuba después de un trabajo de colección de cepas nativas, de evaluaciones en condiciones de laboratorio e invernadero, de ensayos de evaluación y validación de las mejores cepas en campos de agricultores en las tres regiones más productoras de frijol, se llegó a la producción de inoculantes. En las provincias de Matanzas y Holguín, se tuvieron las mejores respuestas a la inoculación. Informan que en 1988 se utilizaron tres toneladas de inoculante en frijol común, cantidad que aumentó en 1992-93 a 60 toneladas, lo cual permitió ahorrar 2,7 millones de dólares al país, debido a la reducción de 16000 toneladas de urea en la producción de frijol.

Durante varios años, el Programa Cooperativo Regional de Frijol para Centroamérica, México y El Caribe (PROFRIJOL), llevó a cabo un proyecto sobre fijación biológica del nitrógeno, donde se dio énfasis a la selección de cepas de Rhizobium y la interacción cepacultivar. Los resultados obtenidos en diferentes localidades y años en la región Centroamericana, mostraron una respuesta positiva del frijol, a la inoculación con un grupo de cepas. Con la mezcla de cepas de mayor consistencia en incrementar los rendimientos de la leguminosa, se inició la producción de inoculante comercial en Costa Rica; las primeras evaluaciones en campo de es- te inoculante, mostraron una respuesta positiva (Acuña, 1997). Por lo anterior, el Proyecto PROFRIJOL que opera con fondos del Gobierno Suizo a través de la Cooperación Suiza para el Desarrollo (COSUDE), decidió realizar validaciones técnicas sobre el uso de este inoculante en frijol en cuatro países de la región Centroamericana, con el propósito de recabar mayor información en campos de agricultores sobre esta tecnología en la producción comercial de grano de frijol.

\section{MATERIALES Y MÉTODOS}

El ensayo regional se programó para sembrarse en 45 sitios distribuidos en Costa Rica (20), Panamá (10), Nicaragua (10) y El Salvador (5) durante los ciclos agrícolas de Primera (mayo-agosto), Postrera (septiembre a enero) y Apante (diciembre a marzo) en los años de 1998 y 1999. Con base en experiencias previas, se diseñaron cinco tratamientos básicos para ser evaluados en esta validación técnica regional promovida por la Coordinación Regional de PROFRIJOL, con fondos del Gobierno Suizo (COSUDE):
1. Testigo absoluto (sin fertilizante, sin inoculante)
2. Inoculado (sólo Rhizobium, $0,8 \mathrm{~kg} / \mathrm{ha}$ )
3. Fertilizacion intermedia
4. Fertilización completa
5. Testigo del agricultor

El inoculante a base de cepas de Rhizobium previamente seleccionadas por su eficiencia en nodulación y fijación biológica de nitrógeno en la región Centroamericana, fue preparado y distribuido por la Universidad de Costa Rica. La variedad, fertilización utilizada y el manejo agronómico del cultivo en cada sitio de evaluación, fueron los recomendados por la institución nacional en cada caso. Los tratamientos tres y cuatro específicos utilizados en cada uno de los países participantes, fueron como sigue:

\section{Costa Rica, Región Brunca (sur):}

3. Fertilización intermedia (100 kg/ha de 12-24-12) + Inoculante $(0,8 \mathrm{~kg} / \mathrm{ha})$.

4. Fertilización recomendada (125 kg/ha de 12-24-12).

Costa Rica, Región Huetar Norte:

3. Fertilización intermedia (100-150 kg/ha de 12-24$12)+$ Inoculante $(0,8 \mathrm{~kg} / \mathrm{ha})$.

4. Fertilización recomendada (200-300 kg/ha 12-24-12). 
Panamá:

3. Fertilización intermedia (75 kg/ha de 18-46-0) + inoculación $(0,8 \mathrm{~kg} / \mathrm{ha})$.

4. Fertilización recomendada (112 kg de 18-46-0 + 180 $\mathrm{kg}$ de urea)/ha.

Nicaragua:

3. Fertilización intermedia $(60 \mathrm{~kg} / \mathrm{ha}$ de $18-46-0)+$ inoculante.

4. Fertilización recomendada (125 kg/ha de 18-46-0).

El Salvador

3. Fertilización intermedia (45 kg/ha de 16-20-0) + inoculación.

4. Fertilización recomendada (90 kg de 16-20-0).

Para la evaluación de los tratamientos se utilizó un diseño de bloques completos al azar con tres repeticiones por ensayo y una parcela experimental de 50 a 100 $\mathrm{m}^{2}$. Se cuantificó el rendimiento de grano como parámetro principal y también se registraron los costos de producción para el análisis económico respectivo. Para los análisis de varianza se utilizaron los promedios de rendimiento y costos de producción por sitio de prueba, agrupando los ensayos por regiones. En los análisis económicos se utilizó la metodología de presupuesto parcial (análisis marginal). En algunos sitios no se instaló el tratamiento cinco (testigo agricultor), por lo que en el análisis regional esta variante se eliminó.

\section{RESULTADOS Y DISCUSIÓN}

Los resultados en rendimiento de grano de los 39 sitios analizados por regiones, por países y a nivel Centroamericano, mostraron diferencias significativas para tratamientos. En Costa Rica los ensayos se instalaron principalmente entre 1998 y 1999; se obtuvieron resultados en 20 sitios. En tres de las cuatro regiones en que se agruparon los ensayos (Concepción-Veracruz, Chánguena, Guatuso, Chiles), no hubo diferencias significativas en el rendimiento en grano entre los tratamientos evaluados. Cuando se aplicó inoculante y fertilización intermedia, el incremento de los rendimientos fue significativo en dos de las cuatro regiones; este mismo resultado se observó al agrupar los ensayos en dos regiones (Brunca, 12; Huetar, 8) y en el análisis de país (20 ensayos). En todos los casos, el tratamiento tres resultó estadísticamente igual a la fertilización recomendada (Fer Rec). Por lo que respecta al análisis económico utilizando la metodología de presupuesto parcial, en todas las regiones de Costa Rica fue seleccionado el tratamiento dos, en dos regiones el tratamiento tres y en tres ocasiones la variante cuatro (Cuadros 1,2 y 3 ).

Cuadro 1. Rendimientos obtenidos en la validación técnica de inoculantes en frijol en Costa Rica. Promedio de 20 sitios agrupados en cuatro ambientes. PITTA Frijol, PROFRIJOL, 1997-98 y 1998-99.

\begin{tabular}{|c|c|c|c|c|c|}
\hline Tratamiento & $\begin{array}{l}\text { Concepción } \\
\text { Veracruz } \\
\text { (6 } \text { sitios) }\end{array}$ & Chánguena & Guatuso & $\begin{array}{l}\text { Chiles } \\
\text { (4 sitios)( }\end{array}$ & $\begin{array}{l}\text { C. Rica } \\
\text { (20 sitios) }\end{array}$ \\
\hline $\begin{array}{l}\text { Testigo } \\
\text { absoluto }\end{array}$ & $548 b^{*}$ & $752 \mathrm{~b}$ & $634 \mathrm{bc}$ & $858 \mathrm{~b}$ & $688 \mathrm{~b}$ \\
\hline Inoculado & $641 \mathrm{~b}$ & $862 \mathrm{ab}$ & $530 \mathrm{c}$ & $1074 \mathrm{ab}$ & $772 \mathrm{~b}$ \\
\hline $\begin{array}{l}\text { Fertilización } \\
\text { intermedia }\end{array}$ & $882 \mathrm{a}$ & $920 \mathrm{a}$ & $785 \mathrm{ab}$ & $1365 \mathrm{a}$ & $971 \mathrm{a}$ \\
\hline $\begin{array}{l}\text { Fertilización } \\
\text { completa }\end{array}$ & $1038 \mathrm{a}$ & $912 \mathrm{a}$ & $920 \mathrm{a}$ & $1171 \mathrm{ab}$ & $1003 \mathrm{a}$ \\
\hline C.V. $(\%)$ & 22,09 & 11,07 & 14,43 & 19,48 & 19,50 \\
\hline DMS 0,05 & 211 & 117 & 165 & 348 & 106 \\
\hline
\end{tabular}

*Las letras señalan la comparación de promedios utilizando la prueba de Duncan 0,05 .

En Panamá las validaciones técnicas se instalaron en postrera 1998-99. Se obtuvieron datos de nueve sitios y se agruparon en dos regiones para el análisis de los rendimientos. En la región de Caisán (cuatro sitios), la fertilización recomendada obtuvo los mayores rendimientos; los tratamientos tres y dos, se ubicaron como segundos y la menor producción se obtuvo con el testigo absoluto. En la otra región (cinco sitios), no hubo diferencias. A nivel de país, el análisis mostró los mismos resultados de Caisán. El análisis económico de presupuesto parcial a nivel de país, seleccionó los tratamientos dos y tres como de mayor retorno económico (Cuadros 4 y 5 ).

Cuadro 2. Rendimientos obtenidos en la validación técnica de inoculantes en frijol en Costa Rica. Promedio de 20 sitios agrupados en dos ambientes. PITTA Frijol, PROFRIJOL, 1997-98 y 1998-99.

\begin{tabular}{lccc}
\hline Tratamiento & $\begin{array}{c}\text { Brunca } \\
(\mathbf{1 2} \text { sitios })\end{array}$ & $\begin{array}{c}\text { Huetar Norte } \\
(\mathbf{8} \text { sitios })\end{array}$ & $\begin{array}{c}\text { Costa Rica } \\
(\mathbf{2 0} \text { sitios })\end{array}$ \\
\hline $\begin{array}{l}\text { Testigo } \\
\text { absoluto }\end{array}$ & $650 \mathrm{~b}^{*}$ & $745 \mathrm{~b}$ & $688 \mathrm{~b}$ \\
$\begin{array}{l}\text { Inoculado } \\
\text { Fertilización }\end{array}$ & $751 \mathrm{~b}$ & $802 \mathrm{~b}$ & $772 \mathrm{~b}$ \\
$\begin{array}{l}\text { intermedia } \\
\text { Fertilización }\end{array}$ & $901 \mathrm{a}$ & $1075 \mathrm{a}$ & $971 \mathrm{a}$ \\
$\begin{array}{l}\text { completa } \\
\text { C.V. (\%) }\end{array}$ & 19,21 & $1046 \mathrm{a}$ & $1003 \mathrm{a}$ \\
DMS 0,05 & 131 & 20,45 & 19,50 \\
& & 195 & 106 \\
\hline
\end{tabular}

*Las letras señalan la comparación de promedios utilizando la prueba de Duncan 0,05. 
Cuadro 3. Resultados en beneficios netos (US\$/ha) en la validación técnica de inoculantes en frijol en Costa Rica. Promedio de 17 sitios agrupados en seis ambientes. PITTA Frijol, PROFRIJOL, 1997-98 y 1998-99.

\begin{tabular}{|c|c|c|c|c|c|c|c|}
\hline Tratamiento & $\begin{array}{l}\text { Chánguena } \\
\text { (2 sitios) }\end{array}$ & $\begin{array}{l}\text { Concepción/Veracruz } \\
\text { (2 sitios) }\end{array}$ & $\begin{array}{l}\text { Chánguena } \\
\text { (2 sitios) }\end{array}$ & $\begin{array}{c}\text { Concepción/Veracruz } \\
\text { (4 sitios) }\end{array}$ & $\begin{array}{c}\text { Chiles } \\
\text { (4 sitios) }\end{array}$ & $\begin{array}{l}\text { Upala } \\
\text { (3 sitios) }\end{array}$ & $\begin{array}{l}\text { Costa Rica } \\
(6 \text { ambientes })^{1}\end{array}$ \\
\hline Testigo absoluto & 748 & 429 & 166 & 420 & 260 & 543 & $428 b^{* *}$ \\
\hline Inoculado & $806 *$ & $455 *$ & $311 *$ & $433 *$ & $500 *$ & $558 *$ & $511 \mathrm{ab}$ \\
\hline Ferlilización intermedia & 776 & 705 & $303 *$ & 401 & $501 *$ & 535 & $537 \mathrm{a}$ \\
\hline Fertilización completa & 806 & $783 *$ & $376 *$ & $601 *$ & 456 & 531 & 592 a \\
\hline C.V. $(\%)$ & & & & & & & 15,92 \\
\hline DMS 0,05 & & & & & & & 101 \\
\hline
\end{tabular}

* Tratamientos seleccionados en análisis económico de presupuesto parcial.

${ }^{1}$ Análisis de varianza considerando como repeticiones los beneficios netos promedio por ambiente.

** Rango en base a Duncan 0,05\%.

En Nicaragua se instalaron los ensayos en las épocas de Primera, Postrera y Apante. De Primera sólo se lograron resultados en Carazo y de Postrera se perdieron los tres instalados por efectos del huracán Mitch; en Apante se instalaron ocho ensayos. El análisis incluyó seis ensayos para rendimiento de grano, agrupados en dos regiones. En Nueva Guinea (cuatro sitios), los tratamientos tres y cuatro, mostraron rendimientos superiores al testigo absoluto; los tratamientos dos y tres, fueron iguales entre sí. En la otra región (dos sitios), fueron iguales y superiores los tratamientos tres y cuatro. A nivel de país, también los tratamientos tres y cuatro fueron de mayor rendimiento. En el análisis económico se incluyeron ocho sitios; los tratamientos seleccionados en las tres regiones en que se agruparon los ensayos (Carazo, Matagalpa y Nueva Guinea), fueron tres y cuatro; el tratamiento dos también fue seleccionado en la región de Nueva Guinea (Cuadros 6 y 7).

Cuadro 4. Resultados en rendimiento $(\mathrm{kg} / \mathrm{ha})$ en la validación técnica de inoculantes en frijol en Panamá. Promedio de nueve sitios agrupados en dos ambientes. IDIAP, PROFRIJOL, 1998-99.

\begin{tabular}{|c|c|c|c|}
\hline Tratamiento & $\begin{array}{l}\text { Caisán } \\
\text { (4 sitios) }\end{array}$ & $\begin{array}{c}\text { Otros } \\
\text { (5 sitios) }\end{array}$ & $\begin{array}{l}\text { Panamá } \\
\text { (9 sitios) }\end{array}$ \\
\hline $\begin{array}{l}\text { Testigo } \\
\text { absoulto }\end{array}$ & $2085 \mathrm{c}^{*}$ & $2033 \mathrm{~b}$ & $2085 \mathrm{c}$ \\
\hline Inoculado & $2423 \mathrm{~b}$ & $2301 \mathrm{ab}$ & $2433 \mathrm{~b}$ \\
\hline $\begin{array}{l}\text { Fertilización } \\
\text { intermedia }\end{array}$ & $2499 \mathrm{~b}$ & $2440 a b$ & $2499 \mathrm{~b}$ \\
\hline $\begin{array}{l}\text { Fertilización } \\
\text { completa }\end{array}$ & 2902 a & $2712 \mathrm{a}$ & 2902 a \\
\hline C.V. $(\%)$ & 10,74 & 17,55 & 14,01 \\
\hline DMS 0,05 & 437 & 573 & 338 \\
\hline
\end{tabular}

*Las letras señalan la comparación de promedios utilizando la prueba de Duncan 0,05.

En El Salvador se obtuvieron resultados de cuatro ensayos. A nivel de promedios, el mayor rendimiento lo obtuvo el tratamiento tres (Fer Par $+\mathrm{Rh}$ ); en segundo
Cuadro 5. Resultados en rendimiento y beneficios netos en la validación técnica de inoculantes en frijol en Panamá. Promedio de nueve sitios. IDIAP, PROFRIJOL, 1998-99.

\begin{tabular}{|c|c|c|}
\hline Tratamiento & $\begin{array}{c}\text { Rendimiento (D) } \\
(\mathrm{kg} / \mathrm{ha})\end{array}$ & $\begin{array}{l}\text { Beneficio neto } \\
\text { (US\$) }\end{array}$ \\
\hline $\begin{array}{l}\text { Testigo } \\
\text { absoluto }\end{array}$ & $2085 \mathrm{c}^{*}$ & 1452 \\
\hline Inoculado & $2423 \mathrm{~b}$ & $1527 * *$ \\
\hline $\begin{array}{l}\text { Fertilización } \\
\text { intermedia }\end{array}$ & $2499 \mathrm{~b}$ & $1743 * *$ \\
\hline $\begin{array}{l}\text { Fertilización } \\
\text { completa }\end{array}$ & 2902 a & 1561 \\
\hline C.V. $(\%)$ & 14,01 & \\
\hline DMS 0,05 & 338 & \\
\hline
\end{tabular}

*Las letras señalan la comparación de promedios utilizando la prueba de Duncan 0,05 .

**Tratamientos seleccionados en análisis económico de presupuesto parcial.

lugar se ubicaron los tratamientos dos $(-\mathrm{N}+\mathrm{Rh})$ y cuatro (Fer Rec) con igualdad estadística. El menor rendimiento fue para el testigo absoluto (-N - Rh). Al revisar los rendimientos obtenidos en cada uno de los cuatro sitios de El Salvador, en las localidades de Tonacatepeque y San Juan Opico, el tratamiento de fertilización parcial más inoculación (Fer Par $+\mathrm{Rh}$ ), fue superior al resto de los tratamientos; este resultado inesperado se vio reflejado en el promedio a nivel de país. Es muy probable que por azar el tratamiento de referencia, en ambos sitios haya estado ubicado en suelo de muy baja fertilidad y/o menor disponibilidad de humedad. No obstante lo anterior, los resultados obtenidos en El Salvador también muestran la bondad del Rhizobium y especialmente la combinación de un poco de fertilizante más inoculante. En este país no se obtuvieron los datos para el análisis económico (Cuadro 8).

En la Región Centroamericana, el análisis estadístico del rendimiento de 39 sitios, mostró como mejores por rendimiento de grano a los tratamientos tres y cua- 
Cuadro 6. Rendimientos obtenidos en la validación técnica de inoculantes en frijol en Nicaragua. Promedio de seis sitios agrupados en dos ambientes. INTA, PROFRIJOL, 1998-99.

\begin{tabular}{lccc}
\hline Tratamiento & $\begin{array}{c}\text { Nueva Guinea } \\
\text { (4 sitios) }\end{array}$ & $\begin{array}{c}\text { Otros } \\
(\mathbf{2} \text { sitios })\end{array}$ & $\begin{array}{l}\text { Nicaragua } \\
(6 \text { sitios })\end{array}$ \\
\hline $\begin{array}{l}\text { Testigo } \\
\text { absoluto }\end{array}$ & $696 \mathrm{~b}^{*}$ & $815 \mathrm{~b}$ & $736 \mathrm{~b}$ \\
$\begin{array}{l}\text { Inoculado } \\
\begin{array}{l}\text { Fertilización } \\
\text { intermedia }\end{array}\end{array}$ & $747 \mathrm{ab}$ & $790 \mathrm{~b}$ & $762 \mathrm{~b}$ \\
$\begin{array}{l}\text { Fertilización } \\
\text { completa }\end{array}$ & $889 \mathrm{a}$ & $883 \mathrm{a}$ & $887 \mathrm{a}$ \\
$\begin{array}{l}\text { C.V. (\%) } \\
\text { DMS 0,05 }\end{array}$ & 12,26 & $885 \mathrm{a}$ & $888 \mathrm{a}$ \\
\hline
\end{tabular}

*Las letras señalan la comparación de promedios utilizando la prueba de Duncan 0,05.

tro. En segundo nivel, se ubicó el tratamiento dos y por último el tratamiento uno. Los análisis económicos de presupuesto parcial realizados en 34 ensayos agrupados en 10 ambientes, seleccionaron en ocho ocasiones al tratamiento dos, en seis ambientes al tratamiento tres y en seis oportunidades al tratamiento cuatro (Cuadros 9 y 10).

Los resultados obtenidos muestran en todos los casos, una tendencia de incremento del rendimiento al usar sólo inoculante; este incremento fue significativo en Panamá y en el análisis de todos los sitios a nivel Centroamérica. Los resultados en rendimiento de grano, están de acuerdo con lo informado en la literatura (Ferrera et al., 1990; Pineda, 1992; Hernández et al., 1994; Acuña, 1997) y confirman la bondad del inoculante utilizado. Estos resultados muestran también que hubo un efecto significativo de los factores del ambien-

Cuadro 7. Resultados en beneficios netos (US\$) en la validación técnica de inoculantes en frijol en Nicaragua. Promedio de ocho sitios agrupados en tres ambientes. INTA, PROFRIJOL, 1998 y 1999.

\begin{tabular}{|c|c|c|c|c|}
\hline \multicolumn{2}{|c|}{$\begin{array}{r}\text { Tratamiento Carazo } \\
\text { (1 sitio) }\end{array}$} & \multirow{2}{*}{$\begin{array}{c}\text { Matagalpa } \\
\text { (3 sitios) } \\
881\end{array}$} & \multirow{2}{*}{$\begin{array}{c}\text { Nueva Guinea } \\
\text { (4 sitios) } \\
626\end{array}$} & \multirow{2}{*}{$\begin{array}{c}\begin{array}{c}\text { Promedio } \\
\text { (3 ambientes) }\end{array} \\
672 \mathrm{~b}^{* *}\end{array}$} \\
\hline $\begin{array}{l}\text { Testigo } \\
\text { absoluto }\end{array}$ & 510 & & & \\
\hline Inoculado & 539 & 870 & $667^{*}$ & $692 \mathrm{~b}$ \\
\hline $\begin{array}{l}\text { Fertilización } \\
\text { intermedia }\end{array}$ & $581 *$ & $931 *$ & $768^{*}$ & $760 \mathrm{a}$ \\
\hline $\begin{array}{l}\text { Fertilización } \\
\text { completa }\end{array}$ & $587 *$ & $915^{*}$ & $750 *$ & $751 \mathrm{a}$ \\
\hline C.V. $(\%)$ & & & & 10,05 \\
\hline DMS 0,05 & & & & 101 \\
\hline
\end{tabular}

* Tratamientos seleccionados en análisis económico de presupuesto parcial. INTA. Informe técnico 1998-99.

${ }^{1}$ Análisis de varianza considerando como repeticiones los beneficios netos promedio por ambiente

** Rango, Duncan 0,05\%. te entre sitios, sobre la capacidad de fijación del Rhizobium como lo señala la literatura (Graham, 1990; Ferrera y Almaraz, 1996), pero evidencian que las cepas con las que se elaboró el inoculante, mostraron ser efectivas, de amplio rango de adaptación y con capacidad de competir con el Rhizobium nativo (Ferrera et al., 1990; Ferrera y Almaraz, 1996).

Cuadro 8. Rendimientos obtenidos en la validación técnica de inoculantes en frijol en El Salvador. Promedio de 4 sitios. CENTA, PROFRIJOL, 1998-99.

\begin{tabular}{lcc}
\hline Tratamiento & Rendimiento (kg/ha) & Rango (Duncan 0,05) \\
\hline Testigo absoluto & 512 & $\mathrm{c}^{*}$ \\
Inoculado & 721 & $\mathrm{~b} \mathrm{c}$ \\
Fertilización intermedia & 1156 & $\mathrm{a}$ \\
Fertilización completa & 893 & $\mathrm{~b}$ \\
C.V. $(\%)$ & 16,22 & \\
DMS 0,05 & 213 & \\
\hline
\end{tabular}

*Las letras señalan la comparación de promedios utilizando la prueba de Duncan 0,05.

Cuando se consideró el análisis económico de presupuesto parcial, el tratamiento que llevó sólo inoculante y el menor costo de inversión entre los que utilizaron insumos, resultó seleccionado en el $80 \%$ de los casos a nivel centroamericano. De acuerdo con los resultados en rendimiento y del análisis económico, el uso de inoculante elaborado con las cepas de PROFRIJOL, constituye una opción de baja inversión para incrementar los rendimientos de frijol en la región centroamericana. Los países deberán especificar los sitios en donde deberá utilizarse esta tecnología.

Cuadro 9. Resultados promedio de rendimiento de grano $(\mathrm{kg} / \mathrm{ha})$ y beneficios netos (US\$/ha) en la validación técnica de inoculantes en frijol en cuatro países de Centroamérica. PROFRIJOL, 1997-98 y 1998-99

\begin{tabular}{lcc}
\hline Tratamiento & $\begin{array}{c}\text { Rendimiento } \\
(\mathbf{k g} / \mathbf{h a})\end{array}$ & $\begin{array}{c}\text { Beneficios netos } \\
\text { (US\$/ha) }\end{array}$ \\
\hline 1. (-N -Rh) & $1000 \mathrm{c}$ & $603 \mathrm{c}^{* *}$ \\
2. (-N +Rh) & $1146 \mathrm{~b}$ & $667 \mathrm{bc}$ \\
3. (Fer Par +F) & $1330 \mathrm{a}$ & $724 \mathrm{ab}$ \\
4. (Fer Rec) & $1412 \mathrm{a}$ & $737 \mathrm{a}$ \\
C.V. $(\%)$ & 19,07 & 10,55 \\
DMS 0,05 & 104 & 66
\end{tabular}

* Las literales en todos los casos señalan la comparación de promedios utilizando la prueba de Duncan 0,05.

1 Análisis de varianza considerando como repeticiones como repeticiones los beneficios netos promedio por ambiente.

Cuando además de inocular se aplicó fertilización intermedia, en todos los casos con excepción de uno de los ambientes de Panamá, los rendimientos fueron sig- 
Cuadro 10. Resumen de los resultados del análisis económico por dominancia parcial por regiones dentro de países en Centroamérica, en la validación de inoculantes en frijol. PROFRIJOL, 1997-98 y 1998-99.

\begin{tabular}{|c|c|c|c|c|c|c|c|c|c|c|}
\hline Tratamiento & $\begin{array}{l}\text { Chánguena } 1 \\
\text { (2 sitios) }\end{array}$ & $\begin{array}{l}\text { Concepción/ } \\
\text { Veracruz } \\
\text { (2 sitios) }\end{array}$ & $\begin{array}{c}\text { Chánguena } 2 \\
\text { (2 sitios) }\end{array}$ & $\begin{array}{l}\text { Concepción/ } \\
\text { Veracruz } \\
\text { (4 sitios) }\end{array}$ & $\begin{array}{l}\text { / Los Chiles } \\
\text { (4 sitios) }\end{array}$ & $\begin{array}{l}\text { Upala } \\
\text { (3 sitios) }\end{array}$ & $\begin{array}{l}\text { Panama } \\
\text { (9 sitios) }\end{array}$ & $\begin{array}{l}\text { Carazo } \\
\text { (1 sitio) }\end{array}$ & $\begin{array}{c}\text { Matagalpa } \\
(3 \text { sitios })\end{array}$ & $\begin{array}{l}\text { Nueva Guinea } \\
\text { (4 sitios) }\end{array}$ \\
\hline Testigo absoluto & 748 & 429 & 166 & 420 & 260 & 543 & 1452 & 510 & 881 & 626 \\
\hline Inoculado & $806^{*}$ & $455^{*}$ & $311^{*}$ & $433^{*}$ & $500 *$ & $558 *$ & $1527 *$ & 539 & 870 & $667 *$ \\
\hline Media fertilización & 776 & 705 & $303 *$ & 401 & $501 *$ & 535 & $1743 *$ & $581 *$ & $931^{*}$ & $768^{*}$ \\
\hline Fertilización completa & 806 & $783^{*}$ & $376^{*}$ & $601 *$ & 456 & 531 & 1561 & $587 *$ & $915^{*}$ & $750 *$ \\
\hline
\end{tabular}

* Tratamientos seleccionados en análisis económico de presupuesto parcial.

nificativamente superiores al testigo sin inocular. En cuatro de las nueve regiones en que se agruparon los ensayos, este tratamiento fue superior al tratamiento inoculado y en ocho de los 10 ambientes, fue igual a la fertilización completa. Estos resultados muestran la necesidad de inocular la semilla con Rhizobium y complementar con fertilizantes químicos, tanto de elementos nutricionales como fósforo, así como la adición de un poco de nitrógeno, si se quiere tener una mayor producción de frijol. Lo encontrado en este ensayo de validación técnica regional, apoya los resultados parciales obtenidos anteriormente en la misma región (Acuña, 1997) y están de acuerdo con López et al. (1984), citados por Ferrera et al. (1990), Acuña et al. (1989) y Beck y Valdez (1994), quienes informan de la necesidad de aplicar fósforo y la posibilidad de utilizar dosis bajas de nitrógeno como complemento a la inoculación.

En el $60 \%$ de los ambientes en que se agruparon los 39 ensayos, fue redituable utilizar fertilización intermedia e inoculación a la semilla. No obstante que el tratamiento tres constituye una alternativa de mayor inversión para incrementar la producción de frijol en relación a la sola inoculación, representa una opción de menor costo al uso de la fertilización completa.

La fertilización completa mostró en general una tendencia de mayores rendimientos; sin embargo, la diferencia observada fue estadísticamente significativa al resto de tratamientos, sólo en la región de Caisán en Panamá. Por beneficio económico, en seis regiones productoras de frijol de las 10 en que se agruparon los ensayos para el análisis económico, el tratamiento con la fertilización completa fue seleccionado. Como lo señala la literatura, estos resultados muestran la bondad de los fertilizantes en la producción de frijol, pero evidencian la existencia de otras opciones igualmente redituables, de menor costo y daño al ambiente. Esta alternativa de mayor inversión de capital, podrá ser utilizada en las regiones donde hubo respuesta a la fertilización completa y por agricultores de mayores recursos económicos.
Los resultados obtenidos en esta validación técnica sobre el uso de Rhizobium para la producción de frijol en la Región Centroamericana, señalan que en las regiones frijoleras tradicionales, es posible seleccionar cepas de Rhizobium eficientes en fijación biológica de nitrógeno. Estos resultados contrastan con la aseveración muy común que menciona casi como imposible lograr incrementos en la producción de frijol mediante el uso de inoculantes a base de Rhizobium, por la existencia de un gran número de cepas nativas altamente competitivas, que anulan el efecto de las cepas introducidas altamente fijadoras de nitrógeno. Es importante señalar que los resultados obtenidos en este trabajo, fueron posibles debido al uso de inoculante elaborado con cepas que fueron seleccionadas en decenas de ensayos en campo, realizados por los países en varios años, regiones y sitios.

\section{CONCLUSIONES}

Los resultados de la validación técnica de inoculantes en frijol con cepas de Rhizobium eficientes en fijación biológica de nitrógeno en cuatro países de Centroamérica, permiten concluir lo siguiente:

El cultivo de frijol muestra respuesta en rendimientos de grano a la inoculación con Rhizobium y/o aplicación de fertilizantes en la Región Centroamericana.

Bajo una perspectiva de baja inversión, el uso de inoculantes a base de cepas eficientes en fijación biológica de nitrógeno, es la mejor opción para agricultores de bajos recursos.

La combinación de una fertilización intermedia y uso de inoculantes, es una opción con mayores niveles de producción, pero con una mayor inversión de capital.

El uso de una fertilización completa, sigue siendo recomendable para la producción de frijol en la Región Centroamericana, pero con niveles de costos mayores al uso de inoculantes o del tratamiento combinado. 
Las conclusiones anteriores derivadas de los resultados obtenidos en la validación técnica realizada, permiten recomendar el uso de inoculante elaborado con las cepas seleccionadas de PROFRIJOL en la Región Centroamericana, ya sea aplicado sólo o en combinación con una fertilización parcial. Esta tecnología deberá utilizarse en los sitios de los países donde hubo respuesta en rendimiento de grano y en donde el análisis económico mostró un buen retorno al capital invertido.

\section{LITERATURA CITADA}

ACUÑA, O.; CORDERO, A. 1989. Efecto de diferentes dosis de molibdeno, fósforo y calcio sobre la nodulación y crecimiento del frijol en un suelo ultisol de Puriscal. Agronomía Costarricense 13 (2):193-196.

ACUÑA, O. 1997. Resultados y avances del trabajo de investigación en Rhizobium en frijol en Costa Rica. In Memoria. XLIII Reunión anual del PCCMCA. Ciudad de Panamá

BECK, D.; VALDEZ, V. 1994. Nitrogen fixation of common bean (Phaseolus vulgaris L.) cultivars at different levels of available phosphorus. In 15th World Congress of Soil Science. Volume 4b. Acapulco, Gro., México.

FERRERA, R.; ALMARÁZ, J. J.; RODRÍGUEZ, M.N.; ESPINOZA, D. 1990. Fijación simbiótica de nitrógeno en frijol. Terra, Vol. 8, Número Especial. p. 35-70.

FERRERA, R.; ALMARÁZ, J. J. 1996. Factores que afectan la fijación simbiótica de nitrógeno en frijol común. In: Pérez, J. y Ferrera, R. eds. Nuevos Horizontes en Agri- cultura: Agroecología y Desarrollo Sostenible. Colegio de Postgraduados en Ciencias Agrícolas. Montecillo, México. p.

GRAHAM, P.; APOLITANO, C.; FERRERA, R.; HALLIDAY, J.; LÉPIZ, R.; MENÉNDEZ, O.; RIOS, R.; SAITO, S.; VITERI, S. 1981. The international bean inoculation trials (IBIT). In: Graham, P.; Harris, S. eds. Biological Nitrogen Fixation. Tecnology for Tropical Agriculture. CIAT, Cali, Colombia.

GRAHAM, P. 1990. Problemas de la nodulación y la fijación de nitrógeno en Phaseolus vulgaris L. Una reevaluación. Terra, Vol. 8, Número Especial. p. 71-82.

HERNÁNDEZ, G.; GONZÁLEZ, A.; TOSCANO, V.; SÁNCHEZ, M.; VÁZQUEZ, H.; GUZMÁN, J.; ALMAGUER, N.; NAVARRO, V. 1994. Production of Rhizobium inoculants for Phaseolus vulgaris in Cuba. In 15th World Congress of Soil Science. Volume 4b. Acapulco, Gro., México.

MORALES, M. 1987. Selección y evaluación de cepas de Rhizobium leguminosarum biovar phaseoli tolerantes al suministro restringido de fósforo. Tesis de maestría. CATIE. Turrialba, C.R. 97p.

PINEDA, P. 1992. Mejoramiento de la fijación biológica del nitrógeno en frijol (Phaseolus vulgaris L.) en el Perú. Documento de trabajo No. 18. CIAT, Cali, Colombia.

ROSAS, J.; BLISS, F. 1986. Mejoramiento de la capacidad de fijación de nitrógeno en frijol común. Ceiba 27:95-104.

TRUJILLO, G. 1990. Producción y estado actual de los inoculantes para leguminosas en México. Terra, Vol. 8, Número Especial. p. 83-90. 